\title{
Mobilization of Patients Suffering from Acute Myocardial Infarction - When Is It Too Early?
}

\author{
Diana Opincariu ${ }^{1,2}$, Roxana Hodas ${ }^{1,2}$ \\ ${ }^{1}$ University of Medicine, Pharmacy, Science and Technology, Târgu Mureș, Romania \\ ${ }^{2}$ Cardiac Critical Care Unit, Clinic of Cardiology, County Clinical Emergency Hospital, Târgu Mureș, Romania
}

\section{ABSTRACT}

The significant raise in the number of patients surviving an acute myocardial infarction (AMI) has increased the burden of morbidity and disability due to acute coronary events. This has led to an increasing awareness on the need to develop specific rehabilitation programs, both during the acute phase, as well as in out-patient settings. In the pre-revascularization era, AMI was treated in bed. Regardless of the known therapeutic benefits of bed rest, there are scarce data regarding a standardized protocol for the early rehabilitation of patients with AMI. An extensive group of AMI patients are still exposed to prolonged immobilization, with current data showing a disparity in the length of post-MI bed rest, with a time of immobilization reported to be from 2 to 12 days and 2 to 28 days. The growing body of evidence on the effects of early cardiac rehabilitation programs following an AMI suggests that early mobilization after the index event could improve the inflammatory response and further modulate the ventricular remodeling process. The timing, duration, and intensity of cardiac mobilization has not yet been established, and further research on the effects of mobilization as early as the first 12 to 24 hours after the acute event could be beneficial for both short- and long-term outcomes, inflammation, and ventricular remodeling with subsequent heart failure.

Keywords: acute myocardial infarction, early mobilization, inflammation, cardiac rehabilitation

\section{ARTICLE HISTORY}

Received: August 7, 2019

Accepted: September 5, 2019

\section{CORRESPONDENCE}

Roxana Hodas

Str. Gheorghe Marinescu nr. 38 540139 Târgu Mureș, Romania Tel: +40265215 551

E-mail: roxana.hodas@yahoo.ro

\section{INTRODUCTION}

The recent decrease in mortality rates associated with acute myocardial infarction (AMI) is mainly due to new developments in prevention strategies, in parallel with an extensive progress in therapeutic revascularization and device therapies. The significant raise in the number of patients surviving an AMI has increased the burden of morbidity and disability due to acute coronary events, especially in the increasingly older population. This has led to an increasing awareness on the need to develop specific rehabilitation programs for patients with AMI, both during the acute phase, as well as in out-patient settings, in order to facilitate patient reintegration from both a physical as well as a psychosocial standpoint. ${ }^{1-3}$

In the pre-revascularization era, AMI was treated in bed. In the 1940 s and 1950s, AMI patients were bedridden for a period as long as 6 weeks, during which it was considered that the injured myocardium would heal and the probability of early complications would decrease. Despite this belief, several complications derived from a prolonged period of bed rest arose, including venous thrombosis and subsequent pulmonary embolism, stasis pneumonia, atelectasis, pressure ulcers, muscular atrophy, and depression. ${ }^{4,5}$ On the other hand, the pathophysiological mecha- 
nism of myocardial infarction derives from an imbalance between the myocardial oxygen demand and supply. The oxygen supply is affected by the impaired coronary flow due to acute coronary occlusion, while the demand is enhanced by increased heart rate, contractility, and workload. Consequently, patient bed rest has become an important method for the reduction of ventricular workload in the acute phase of a myocardial infarction. ${ }^{4,6}$

Regardless of the known therapeutic benefits of bed rest following an AMI, there are scarce data regarding a standardized protocol for the early rehabilitation of patients with AMI. The lack of a guideline consensus for the duration of bed rest has been illustrated by the disparity of the reported immobilization times after AMI in several studies that ranges from 2 days, up to almost one month. ${ }^{4,6}$

\section{STATUS QUO OF CARDIAC REHABILITATION IN THE ACUTE PHASE OF AMI}

After revascularization, patients with ST-segment elevation AMI (STEMI) should be admitted to the Intensive Cardiac Care Unit (ICCU) or an equally equipped unit with possibility for monitorization and specialized care. The medical staff providing the highly specialized care should be trained in the management of acute coronary syndromes and their possible complications, having available invasive and noninvasive monitoring. ${ }^{7,8}$ All AMI patients should be monitored for a minimum of 24 hours after the acute event, with prolongation of this period in case of high risk of arrhythmic complications, hemodynamic instability, severely impaired systolic dysfunction of the left ventricle, unsuccessful reperfusion, or multivessel coronary artery disease. ${ }^{8}$ The European Society of Cardiology (ESC) guidelines for the management of patients with STEMI recommend early mobilization of patients with no complications in case of radial access percutaneous coronary intervention. However, patients with larger infarcts and associated acute heart failure or rhythm disturbances may require prolonged bed rest, mainly until the assessment of cardiac function via noninvasive imaging methods and after stabilization. ${ }^{8}$ The American Heart Association (AHA) guidelines state that STEMI patients without ischemic or heart failure symptoms, and with no mechanical or arrhythmic complication, should not be bedridden for longer than 12 to 24 hours from the onset of symptoms, while patients with hemodynamic instability should be mobilized at the bedside. Patients should also be mobilized early in order to prevent deep vein thrombosis due to prolonged bed rest, for which, if required, the use of prophylactic low molecular weight heparin may be useful. ${ }^{9}$

\section{EARLY VERSUS LATE MOBILIZATION OF AMI PATIENTS STILL A 'CONSENSUS-BASED' PROBLEM}

With almost 50 years of advising bed rest as an inherent management for AMI, few developments have been made with regard to the early mobilization of patients after AMI as the first step of cardiac rehabilitation (CR). This is true even if a decreasing trend was observed over the last years in both the extend of bed rest and the length of hospitalization for AMI patients. ${ }^{4,10}$ According to current ESC and ACC/AHA guidelines for the management of patients with STEMI, mobilization should be performed in the first 12 and 24 hours following an uncomplicated acute AMI (level of evidence C). ${ }^{8,9}$ However, the basis for these indications is unclear, as the quality of most available studies is unsatisfactory, proving to be rather 'consensus-based' than evidence-based. Therefore, we face a situation in which current recommendations for the length of bed rest are not supported by solid evidence, a surprising fact taking into account the large amount of trials comparing the effectiveness of different medications in AMI patients. ${ }^{6}$ A review of existing randomized controlled trials of early versus later mobilization in uncomplicated AMI patients concluded a generally poor quality for most of the studies, as all these trials were conducted a long time before any reporting standards were implemented. Taking this into account, no inferences could be performed regarding the impact of present-day early mobilization on clinical outcomes. ${ }^{4}$ Based on experimental trials conducted prior to the PCI strategies implementation worldwide, a trend towards decreased mortality was reported in AMI patients with early mobilization associated with a $15 \%$ drop in terms of relative risk reduction. ${ }^{11}$ Cortes et al. emphasized an urgent requirement for updated randomized controlled studies assessing the effects of early mobilization in AMI patients treated according to the current therapy guidelines, considering the current data potentially inadequate, biased, and out-of-date in terms of current clinical practice. ${ }^{11}$ Given the limited body of evidence, most of which comes from old studies and is of slight significance to current clinical practice, it is considered impossible nowadays to take a final judgement on whether early mobilization or longer duration of bed rest could be considered safer.

Moreover, there is a lack of evidence in terms of present patterns of clinical practice associated to progressive mobilization of AMI patients through the Phase I cardiac rehabilitation and the benefits of this intervention. ${ }^{11,12} \mathrm{At}$ this moment, there are no available studies describing present clinical practice and patterns of early mobiliza- 
tion after AMI. As the optimal moment for patient mobilization after AMI remains unknown given the existing evidence, in clinical practice hesitation lingers about the early mobilization element of Phase I recovery after AMI. ${ }^{11}$ Therefore, this intervention is practiced and prescribed in different ways and for different lengths of time due to current differences in hospital care worldwide. ${ }^{12,15}$ Regardless of the evolving patterns of care, an extensive group of AMI patients are still exposed to prolonged immobilization, with the results of several published reviews indicating a disparity in the length of post-MI bed rest, with a time of immobilization reported to be from 2 to 12 days and 2 to 28 days. ${ }^{4,6}$ As bed rest is frequently understood as a required condition to enable monitoring, with increased use of telemetric monitoring, prolonged bed rest is no longer mandatory for monitoring purposes. In these terms, telemonitoring could offer an opportunity to compare short bed rest under 12 hours with longer extents in patients with uncomplicated AMI. ${ }^{7}$

In a prospective pilot observational study conducted by Cortez et al. in three centers, results showed that during the first three days in the CCU, most of the AMI patients remain in bed rest, and mobilization takes place nearly 50 hours after the AMI onset. This indicates a failing in guideline recommendations in current practice, as patients are currently late mobilized and spend $70 \%$ of time in bed rest. ${ }^{14}$ This prospective study pointed out early mobilization as a management gap for the secondary prevention in AMI patients. Despite these differences in the time of bed rest, the outcome of early mobilization of AMI patients during CCU stay may be of clinically importance, as several studies have reported early return to work, as well as reduced mortality, depression, and heart rate variations associated with early mobilization. ${ }^{4,10}$ Therefore, early mobilization as a component of Phase I cardiac rehabilitation should be performed as a strategy to improve the functional status of AMI patients, as well as an approach to endorse adherence to Phase II cardiac rehabilitation. Given the increased body of evidence correlated to the negative effects of prolonged immobilization and the general clinical practice acceptance of the notion that Phase I cardiac rehabilitation should start in the first days of hospitalization, steps should be taken to initiate this intervention as soon as possible in the CCU between the first 24 hours and the third day of hospitalization. AMI patient mobilization can be considered a therapeutic intervention that should be progressively administered and intensively monitored. ${ }^{15}$ Nevertheless, this kind of advice is not evidence-based, therefore the ideal moment for AMI patient mobilization will remain indefinite in the lack of further studies. These should also assess the impact on the patients' quality of life, given that we know almost nothing about the effects on this imperative outcome. ${ }^{16}$ It remains clear that a certain minimum time in bed will always be mandatory in the immediate post-infarction period, and that practical necessities will determine how long this is.

\section{EARLY CARDIAC REHABILITATION AND SYSTEMIC INFLAMMATORY RESPONSE FOLLOWING AN AMI}

Myocardial cell necrosis following an AMI triggers a systemic inflammatory response, which is linked to the process of cardiac repair and subsequent fibrosis. In time, this will lead to left ventricular remodeling and heart failure. A series of imaging and laboratory parameters have been associated with an enhanced ventricular remodeling process including the infarct size and inflammatory biomarkers of the extent of the atherosclerotic coronary lesions. Infarct size can be estimated with the use of imaging techniques that include transthoracic echocardiography and cardiac magnetic resonance, but also via laboratory immunoassays that illustrate myocardial necrosis markers - cardiac troponins, myocardial fraction of creatine kinase, myoglobin. ${ }^{17,18}$ Several studies have shown the correlation between infarct size and the extent of the acute systemic inflammatory response. Rajewska-Tabor et al. have shown that patients with more extensive infarctions (evaluated via cardiac magnetic resonance imaging) presented a more intense inflammatory response as illustrated by higher levels of high-sensitivity C-reactive protein (hs-CRP) and leucocyte count..$^{19}$ Another study published in 2018 showed that the inflammatory response evaluated by the circulating levels of interleukin 8 was directly correlated with the infarct size and the degree of microvascular obstruction assessed by CMR, as well as with the rate of major adverse cardiovascular events at 12 months from the index event, even after adjustment for the peak troponin values. ${ }^{20}$ The inflammatory response following an AMI is responsible for myocardial healing. On one hand, an insufficient inflammatory response may lead to cardiac rupture or left ventricular aneurysm, but on the other hand, an overexpressed response can cause a series of short- and longterm complications and impaired patient outcomes. ${ }^{21-23}$ This indicates that systemic inflammation can be a therapeutic target in further studies, which could revolutionize the treatment of patients in the post-infarction period. ${ }^{24,25}$

Moreover, several studies have shown the benefits of physical exercise on inflammation, as depicted by serum levels of several inflammatory biomarkers includ- 
ing C-reactive protein, interleukins, fibrinogen, adhesion molecules, adiponectin, or tumor necrosis factor alpha. ${ }^{26}$ Physical activity has also been postulated to have benefic effects on atherosclerosis and endothelial dysfunction, via its anti-inflammatory properties. ${ }^{27} \mathrm{~A}$ study on patients with stable chronic heart failure showed that after the implementation of a cardiac rehabilitation program based on 30 minutes of physical exercise per day, 5 days a week, for 6 weeks, there was a significant reduction in the serum levels of tumor necrosis factor alpha and soluble tumor necrosis, as well as significantly better functional capacity. ${ }^{28}$ Another study revealed a significant reduction in hs-CRP levels, even after adjustments for statin therapy and weight reduction, which highly suggests the benefic role of physical training and mobilization on decreasing the systemic inflammatory response in cardiovascular patients. ${ }^{29}$ Many studies have also evaluated the effects of cardiac rehabilitation programs on systemic inflammation in subjects following an acute coronary event. A study on 141 AMI patients who underwent coronary revascularization reported that those who followed 6-8 weeks of cardiac rehabilitation, presented lower hs-CRP levels compared to controls, and the rate of recurrence of major adverse cardiac events (reinfarction, rehospitalization, need for revascularization, or death) was also lower in the case group..$^{30}$ Balen et al. have studied the effect of exercise training on acute phase reactants in the early postAMI period. The AMI group followed a cardiac rehabilitation program after $7.08 \pm 1.6$ days following the index event, and the obtained results showed that early exercise training had significantly decreased the levels of hs-CRP and fibrinogen. ${ }^{31}$ In addition to the beneficial effects on inflammation, early cardiac rehabilitation could improve the adverse left ventricular remodeling process following an AMI. Physical exercise after an acute coronary event has been shown to improve global and regional systolic function, regardless of the extent of coronary atherosclerosis, and also induce a favorable course of the left ventricular remodeling process. ${ }^{32-37}$

The growing body of evidence on the effects of early cardiac rehabilitation programs following an AMI suggests that early mobilization after the index event could improve the inflammatory response and further modulate the ventricular remodeling process. The timing, duration, and intensity of cardiac mobilization has not yet been established, and further research on the effects of mobilization as early as the first 12 to 24 hours after the acute event could be beneficial for both short- and long-term outcomes, inflammation, and ventricular remodeling with subsequent heart failure.

\section{CONCLUSIONS}

Taking into consideration the relatively low number of recent studies on the effect of early mobilization following AMI, there is a clear need for new research on the matter, on larger populations. This should be associated, on national level, with the creation of an early rehabilitation program that could improve patient outcomes, reduce the systemic inflammatory response following the acute loss of myocardial cells, and thus reduce the rate of late complications such as left ventricular remodeling and subsequent heart failure. The early mobilization of patients after AMI will not only reduce the complications consequent to prolonged immobilization, but it will improve the psychological burden derived from it as well.

\section{CONFLICT OF INTEREST}

Nothing to declare.

\section{REFERENCES}

1. Zhang $\mathrm{Y}$, Cao H, Jiang $\mathrm{P}$, Tang $\mathrm{H}$. Cardiac rehabilitation in acute myocardial infarction patients after percutaneous coronary intervention. Medicine (Baltimore). 2018;97:e9785. doi: 10.1097/MD.0000000000009785.

2. Piepoli MF, Corrà U, Dendale $\mathrm{P}$, et al. Challenges in secondary prevention after acute myocardial infarction: A call for action. Eur J Prev Cardiol. 2016;23:1994-2006. doi: 10.1177/2047487316663873.

3. Piepoli MF, Corrà U, Benzer W, et al. Secondary prevention through cardiac rehabilitation: from knowledge to implementation. A position paper from the Cardiac Rehabilitation Section of the European Association of Cardiovascular Prevention and Rehabilitation. Eur J Cardiovasc Prev Rehabil. 2010;17:1-17. doi:10.1097/HJR.ob013e3283313592.

4. Lopes JL, Santos JT, Lima SC, Barros ALBL. Mobilization and early hospital discharge for patients with acute myocardial infarction. Acta Paul Enferm. 2008;21:345-550.

5. Winslow EH. Cardiovascular consequences of bed rest. Heart Lung. 1985;14:236-246.

6. Herkner H, Arrich J, Havel C, Mullner M. Bed rest for acute uncomplicated myocardial infarction. Cochrane Database Syst Rev. 2007;2:Cdo03836.

7. Hasin Y, Danchin N, Filippatos GS, et al. Recommendations for the structure, organization, and operation of intensive cardiac care units. Eur Heart J. 2005;26:1676-1682. doi: 10.1093/ eurheartj/ehi202.

8. Ibanez B, James S, Agewall S, et al. 2017 ESC Guidelines for the management of acute myocardial infarction in patients presenting with ST-segment elevation. Eur Heart J. 2017;00:166. doi: 10.1093/eurheartj/ehx393.

9. O'Gara PT, Kushner FG, Ascheim DD, et al. 2013 ACCF/AHA Guideline for the Management of ST-Elevation Myocardial Infarction A Report of the American College of Cardiology Foundation/American Heart Association Task Force on 
Practice Guidelines. Circulation. 2013;127:e362-e425. doi: 10.1161/CIR.ob013e3182742cf6.

10. Cortes O, Villar J, Devereaux P, DiCenso A. Early mobilization for patients following acute myocardial infarction: A systematic review and meta-analysis of experimental studies. Int J Nurs Stud. 2009;46:1496-1504. doi: 10.1016/j.ijnurstu.2009.03.012.

11. Herkner H, Arrich J, Havel C, Müllner M. Bed rest for acute uncomplicated myocardial infarction. Cochrane Database Syst Rev. 2007;2:CD003836. doi: 10.1002/14651858.CD003836. pub2.

12. Herkner H, Thoennissen J, Nikfardjam M, et al. Short versus prolonged bed rest after uncomplicated acute myocardial infarction: a systematic review and meta-analysis. J Clin Epidemiol. 2003;56:775-781. doi: 10.1016/s08954356(03)00121-5.

13. Vavalle JP, Lopes RD, Chen AY, et al. Hospital length of stay in patients with non-ST-segment elevation myocardial infarction. Am J Med. 2012;125:1085-1094. doi: 10.1016/j. amjmed.2012.04.038.

14. Cortés OL, DiCenso A, McKelvie R. Mobilization patterns of patients after an acute myocardial infarction: a pilot study. Clin Nurs Res. 2015;24:139-155. doi: 10.1177/1054773813508132.

15. Cortés O, Arthur HM. Determinants of referral to cardiac rehabilitation programs in patients with coronary artery disease: a systematic review. Am Heart J. 2006;151:249-256. doi: 10.1016/j.ahj.2005.03.034

16. Belle L, Labarère J, Fourny $\mathrm{M}$, et al. Quality of care for myocardial infarction at academic and nonacademic hospitals. Am J Med. 2012;125:365-373. doi: 10.1016/j.amjmed.2011.11.015.

17. Expert Group on Biomarkers. Biomarkers in Cardiology Part 2: In Coronary Heart Disease, Valve Disease and Special Situations. Arq Bras Cardiol. 2015;104:337-346. doi: 10.5935/ abc.20150061.

18. Mythili S, Malathi N. Diagnostic markers of acute myocardial infarction. Biomed Rep. 2015;3:743-748. doi: 10.3892/ br.2015.500.

19. Rajewska-Tabor J, Pyda M, Kociemba A, Janus M, Lanocha M, Siniawski A. Influence of inflammatory response on infarct size and microvascular obstruction estimated by cardiac magnetic resonance in patients with ST-elevation myocardial infarction. J Cardiovasc Magn Reson. 2015;17:P160. doi: 10.1186/1532-429X-17-S1-P160.

20. Shetelig C, Limalanathan S, Hoffmann P, et al. Association of IL-8 with Infarct Size and Clinical Outcomes in Patients with STEMI. J Am Coll Cardiol. 2018;72:187-198. doi: 10.1016/j. jacc.2018.04.053.

21. Fang L, Moore XL, Dart AM, Wang LM. Systemic inflammatory response following acute myocardial infarction. J Geriatr Cardiol. 2015;12:305-312. doi: 10.11909/j.issn.16715411.2015.03.020.

22. Prabhu SD, Frangogiannis NG. The Biological Basis for Cardiac Repair After Myocardial Infarction: From Inflammation to Fibrosis. Circ Res. 2016;119:91-112. doi: 10.1161/ CIRCRESAHA.116.303577.

23. Ong SB, Hernández-Reséndiz S, Crespo-Avilan GE, et al. Inflammation following acute myocardial infarction:
Multiple players, dynamic roles, and novel therapeutic opportunities. Pharmacol Ther. 2018;186:73-87. doi:10.1016/j. pharmthera.2018.01.001.

24. Saxena A, Russo I, Frangogiannis NG. Inflammation as a therapeutic target in myocardial infarction: learning from past failures to meet future challenges. Transl Res. 2016;167:152166. doi: 10.1016/j.trsl.2015.07.002.

25. Huang S, Frangogiannis NG. Anti-inflammatory therapies in myocardial infarction: failures, hopes and challenges. $\mathrm{Br} \mathrm{J}$ Pharmacol. 2018;175:1377-1400. doi: 10.1111/bph.14155.

26. Beavers KM, Brinkley TE, Nicklas BJ. Effect of exercise training on chronic inflammation. Clin Chim Acta. 2010;411:785-793. doi: 10.1016/j.cca.2010.02.069.

27. Yang J, Cao RY, Gao R, Mi Q, Dai Q Zhu F. Physical Exercise Is a Potential "Medicine" for Atherosclerosis. Adv Exp Med Biol. 2017;999:269-286. doi: 10.1007/978-981-10-4307-9_15.

28. LeMaitre JP, Harris S, Fox KA, Denvir M. Change in circulating cytokines after 2 forms of exercise training in chronic stable heart failure. Am Heart J. 2004;147:100-105. doi: 10.1016/j. ahj.2003.07.001.

29. Biasucci LM. C-Reactive Protein and secondary prevention of coronary events. Clin Chim Acta. 2001;311:49-52. doi: 10.1016/ s0009-8981(01)00558-7.

30. Kim C, Kim DY, Moon CJ. Prognostic influences of cardiac rehabilitation in Korean acute myocardial infarction patients. Ann Rehabil Med. 2011;35:375-380. doi: 10.5535/ arm.2011.35.3.375.

31. Balen S, Vukelić-Damijani N, Persić V, et al. Anti-inflammatory effects of exercise training in the early period after myocardial infarction. Coll Antropol. 2008;32:285-291.

32. Lawler PR, Filion KB, Eisenberg MJ. Efficacy of exercise-based cardiac rehabilitation post-myocardial infarction: a systematic review and meta-analysis of randomized controlled trials. Am Heart J. 2011;162:571-584.e2. doi: 10.1016/j.ahj.2011.07.017.

33. Naughton J, Dorn J, Oberman A, Gorman PA, Cleary P. Maximal exercise systolic pressure, exercise training, and mortality in myocardial infarction patients. Am J Cardiol. 2000;85:416420. doi: 10.1016/s0002-9149(99)00765-1.

34. Garza MA, Wason EA, Zhang JQ. Cardiac remodeling and physical training post myocardial infarction. World J Cardiol. 2015;7:52-64. doi: 10.4330/wjc.v7.i2.52.

35. Zhang YM, Lu Y, Tang Y, et al. The effects of different initiation time of exercise training on left ventricular remodeling and cardiopulmonary rehabilitation in patients with left ventricular dysfunction after myocardial infarction. Disabil Rehabil. 2016;38:268-726. doi: 10.3109/09638288.2015.1036174.

36. Haykowsky M, Scott J, Esch B, et al. A meta-analysis of the effects of exercise training on left ventricular remodeling following myocardial infarction: start early and go longer for greatest exercise benefits on remodeling. Trials. 2011;12:92. doi: 10.1186/1745-6215-12-92.

37. Zhang H, Chang R. Effects of Exercise after Percutaneous Coronary Intervention on Cardiac Function and Cardiovascular Adverse Events in Patients with Coronary Heart Disease: Systematic Review and Meta-Analysis. J Sports Sci Med. 2019;18:213-222. 